\title{
Peptídeo Natriurético Tipo-B e Doenças Cardiovasculares
}

\author{
Luís Beck da Silva, Carlos A. Ferreira, Celso Blacher, Paulo Leães, Haissam Haddad \\ Porto Alegre, RS / Ottawa, ON - Canadá
}

\begin{abstract}
"Cardiologistas e internistas têm agora uma ferramenta para determinar se o paciente tem insuficiência cardíaca elou avaliar sua severidade, assim como os médicos, rotineiramente, solicitam creatinina sérica para determinar se o paciente tem doença renal ou testes de função hepática em pacientes com doenças do fígado."
\end{abstract}

\section{K.L. Baughman, Johns Hopkins}

A descoberta de uma ligação endocrinológica entre o coração e os rins baseia-se em achados de microscopia eletrônica em que células de músculo estriado dos átrios de mamíferos se diferenciam tanto em células contráteis como em células endócrinas. O fator natriurético atrial (FNA), um peptídeo circulante com propriedades natriuréticas, diuréticas e vasodilatadoras, foi descoberto em 1980 por A. J. de Bold, e considerado, na ocasião, como a ligação hormonal entre o coração e os rins. Desde então, um imenso número de investigações multidisciplinares tem sido conduzido para esclarecer o real papel deste peptídeo na patogênese das doenças cardiovasculares, na regulação da pressão arterial e na excreção de sal e água. Essas investigações acabaram se tornando um excelente exemplo de pesquisa evoluída da bancada do laboratório à beira do leito do paciente. Hoje, o peptídeo natriurético tipo-B (PNB) vem gradual e lentamente se estabelecendo na área clínica.

O objetivo deste artigo é prover o clínico de uma revisão atualizada dos progressos ocorridos até o momento, a fim de incluir o peptídeo natriurético tipo-B na prática clínica diária.

\section{Os peptídeos natriuréticos}

FNA, PNB (também chamado de peptídeo natriurético cerebral), e o peptídeo natriurético tipo $\mathrm{C}(\mathrm{PNC})$ constituem

University of Ottawa Heart Institute e Hospital São Francisco, Santa Casa de Porto Alegre - Correspondência: Haissam Haddad - Programa de Insuficiência Cardíaca - Instituto do Coração da Universidade de Ottawa - 40 Ruskin Street Suite H147 - Ottawa - ON, K1Y 4W7 - Canadá -e-mail: hhaddad@ottawaheart.ca Recebido para publicação em 14/10/02

Aceito em 10/4/03 a família dos peptídeos natriuréticos². Seu papel principal é a participação na homeostase cardiovascular e na modulação do crescimento celular. O RNAm de fator natriurético atrial foi encontrado em muitos tecidos, mas é particularmente abundante nos átrios cardíacos. O peptídeo natriurético tipo-B foi, primeiramente, isolado de homogenados de cérebro, mas tambémé encontrado na circulação periférica. No entanto, sua maior concentração encontra-se no tecido miocárdico. Tanto o fator natriurético atrial como o peptídeo natriurético tipo-B são produzidos, normalmente, pelas células de músculo atrial, de onde são liberados. Entretanto, sob condições atípicas, como a doença estrutural miocárdica, o peptídeo natriurético tipo-B parece ser produzido em maior escala pelos ventrículos ${ }^{3}$.

Os peptídeos natriuréticos, em conjunto, contrabalançam os efeitos do sistema renina-angiotensina-aldosterona (SRAA). As concentrações plasmáticas de FNA e peptídeo natriurético tipo-B aumentam em resposta à distensão do tecido atrial e parecem ser antagonistas dos efeitos da angiotensina II no tônus vascular, na secreção de aldosterona, na reabsorção de sódio e no crescimento celular vascular. $\mathrm{O}$ peptídio natriurético tipo-C é encontrado, predominantemente, no cérebro e em células endoteliais e as concentrações no plasma são muito baixas ${ }^{4}$. Por isto, os peptídeos natriuréticos mais amplamente estudados em doenças cardiovasculares são FNA e peptídeo natriurético tipo-B (fig. 1).

\section{Peptídeo natriurético tipo-B como ferramenta diagnóstica}

O peptídeo natriurético tipo-B tem-se mostrado como uma ferramenta diagnóstica confiável na sala de emergência, como teste de rastreamento para pacientes com queixas de dispnéia.

Davis e cols. ${ }^{5}$, após medirem fator natriurético atrial e peptídeo natriurético tipo-B em 52 pacientes com dispnéia na sala de emergência, demonstraram que o peptídeo natriurético tipo-B plasmático à admissão indicou o diagnóstico correto com mais acurácia que a fração de ejeção do ventrículo esquerdo e o fator natriurético atrial. Dao e cols. ${ }^{6}$ mediram o peptídeo natriurético tipo-B em 250 pacientes que se apresentaram à sala de emergência com dispnéia, como prin- 


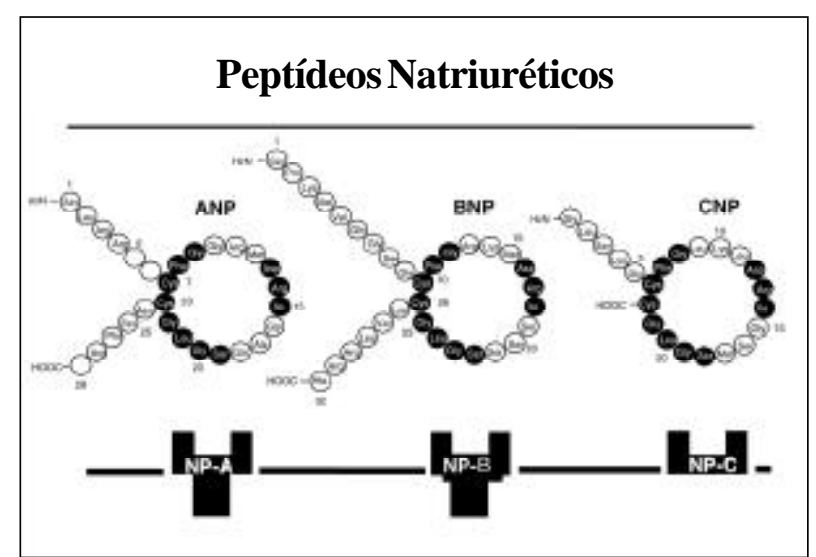

Fig. 1 - A família dos peptídeos natriuréticos. (Copyright Biosite Diagnostics, 2001)

cipal queixa, e encontraram uma significativa discrepância entre os valores de peptídeo natriurético tipo-B em pacientes com e sem o diagnóstico de insuficiência cardíaca. Nesse estudo, peptídeo natriurético tipo-B, no valor de 80pg/ $\mathrm{ml}$, foi altamente sensível e específico para o diagnóstico de insuficiência cardíaca. $\mathrm{O}$ valor preditivo negativo de um peptídeo natriurético tipo-B $<80 \mathrm{pg} / \mathrm{ml}$ foi de $98 \%$, excluindo insuficiência cardíaca (fig. 2).

Mais recentemente, Harrison e cols. ${ }^{7}$ investigaram se o peptídeo natriurético tipo-B de pacientes que chegam à sala de emergência com dispnéia aguda, poderia ser um preditor de futuros eventos cardíacos. Esses autores relataram que um peptídeo natriurético tipo-B $>480 \mathrm{pg} / \mathrm{ml}$ teria sensibilidade de $68 \%$, especificidade de $88 \%$ e acurácia diagnóstica de $85 \%$ em predizer subsequente desfecho de insuficiência cardíaca em 6 meses. Por outro lado, pacientes com peptídeo natriurético tipo-B $<230 \mathrm{pg} / \mathrm{ml}$ apresentavam, no mesmo período, excelente prognóstico com incidência de insuficiência cardíaca de apenas $2.5 \%$. Finalmente, os autores concluíram que, nessa população de pacientes com dispnéia aguda, os valores de peptídeo natriurético tipo-B poderiam ser altamente preditivos de eventos cardíacos em 6 meses.

Um grande ensaio clínico multicêntrico, recentemente completado, examinou 1586 pacientes com dispnéia aguda

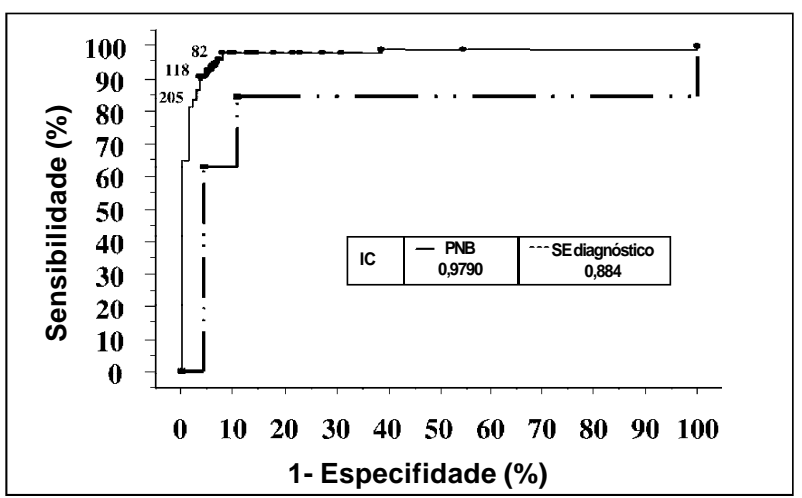

Fig. 2 - Curvas ROC (Receiver operated characteristics) para o peptídeo natriurético tipo-B e para o diagnóstico clínico de insuficiência cardíaca na sala de emergência. (Adaptado de Dao e cols. ${ }^{6}$ ) para testar a capacidade do peptídeo natriurético tipo-B de diferenciar pacientes com insuficiência cardíaca de pacientes com outras causas de falta de ar. Os resultados finais demonstraram que peptídeo natriurético tipo-B teve boa especificidade e alto valor preditivo negativo para excluir o diagnóstico de insuficiência cardíaca, com uma área sob a curva ROC (receiver operating characteristic) de 0.91 . O estudo também confirmou a associação entre os níveis plasmáticos de peptídeo natriurético tipo-B e a severidade de insuficiência cardíaca, segundo a classificação funcional da $\mathrm{New}$ York Heart Association (NYHA) (fig. 3). O mesmo estudo demonstrou que uma simples medida de peptídeo natriurético tipo-B era mais acurada para o diagnóstico de insuficiência cardíaca que os critérios de Framingham de insuficiência cardíaca e a escala do Exame Nacional de Saúde e Nutrição nos Estados Unidos (National Health and Nutrition Examination - NHANES $)^{8}$.

Curvas ROC sugerem que um ponto de corte de peptídeo natriurético tipo-B em $100 \mathrm{pg} / \mathrm{ml}$ confere uma razoável habilidade de discernir pacientes com ou sem insuficiência cardíaca, com uma sensibilidade de $90 \%$, uma especificidade de $76 \%$ e uma acurácia de $83 \%{ }^{9}$.

\section{Peptídeo natriurético tipo-B e o diagnóstico de disfunção diastólica}

Estima-se que 40 a $50 \%$ dos pacientes com diagnóstico de insuficiência cardíaca têm função sistólica preservada, implicando em disfunção diastólica como o diagnóstico mais provável para essa condição. A prevalência de insuficiência cardíaca por disfunção diastólica aumenta com a idade, com uma incidência aproximada de 15 a 25\% em pacientes com menos de 60 anos, 35 a $40 \%$ naqueles entre 60 e 70 anos e $50 \%$ nos acima de $70 \operatorname{anos}^{10}$. O diagnóstico de insuficiência cardíaca por disfunção diastólica geralmente não pode ser distinguido de disfunção sistólica, apenas por meio de história e exame físico, radiografia de tórax e eletrocardiograma ${ }^{11}$. Clinicamente, o diagnóstico de desempenho diastólico anormal é baseado em exclusões, como por exem-

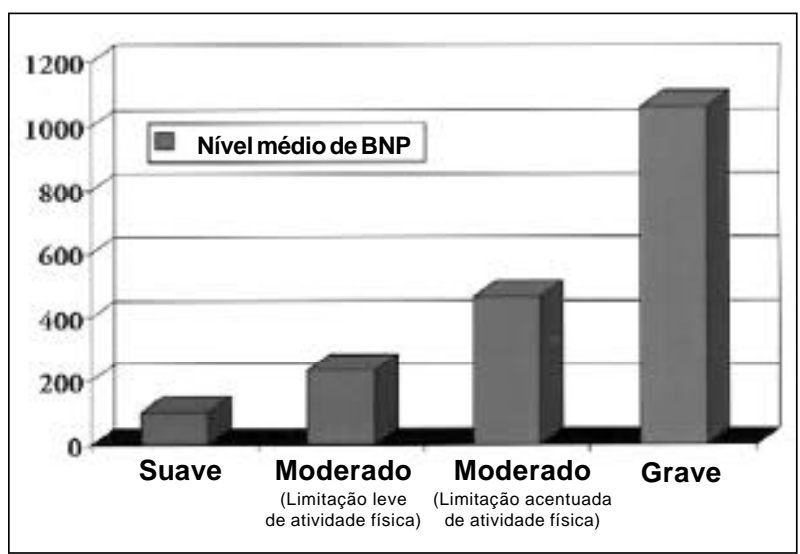

Fig. 3 - Relação entre peptídeo natriurético tipo-B e severidade de insuficiência cardíaca estimada pela classificação funcional de New York Heart Association. (Copyright Biosite Diagnostics, 2001) 
plo ausência de disfunção sistólica em pacientes com insuficiência cardíaca.

Krishnaswamy e cols. ${ }^{12}$ estudaram a utilidade do peptídeo natriurético tipo-B em diagnosticar disfunção diastólica, além da presença de disfunção sistólica. As medidas da fração de ejeção foram avaliadas em 400 pacientes. Em 147, a função ventricular foi considerada normal e os níveis de peptídeo natriurético tipo-B baixos, de $30 \pm 36 \mathrm{pg} / \mathrm{ml}$, enquanto naqueles com disfunção sistólica o peptídeo natriurético tipo-B foi de $416 \pm 413 \mathrm{pg} / \mathrm{ml}$. Pacientes com função sistólica preservada, mas com disfunção diastólica, tiveram valores de $391 \pm 89 \mathrm{pg} / \mathrm{ml}$ ( $<<0.001$ comparados aos normais). A área sob a curva ROC para o peptídeo natriurético tipo-B detectar disfunção diastólica à ecocardiografia em pacientes com insuficiência cardíaca e função sistólica normal foi de 0.958 e para detectar qualquer anormalidade ecocardiográfica de $0.95^{12}$. Outros autores têm também relatado níveis elevados de peptídeo natriurético tipo-B em presença de disfunção diastólica ${ }^{13,14}$.

Mais recentemente, Lubien e cols. ${ }^{15}$ estudaram 294 pacientes encaminhados para avaliação da função ventricular pela ecocardiografia, a fim de diferenciar os vários padrões de enchimento ventricular esquerdo detectados pelas leituras de Doppler em indivíduos com função sistólica normal. Foram excluídos pacientes com disfunção sistólica. Pacientes diagnosticados com qualquer evidência de disfunção diastólica tiveram concentração de peptídeo natriurético tipo-B de $286 \pm 31 \mathrm{pg} / \mathrm{ml}$, enquanto aqueles com ventrículo esquerdo normal tiveram peptídeo natriurético tipo-B de $33 \pm 3 \mathrm{pg} / \mathrm{ml}$. Pacientes com padrão de enchimento tipo restritivo à ecocardiografia apresentaram os mais altos níveis de peptídeo natriurético tipo-B $(408 \pm 66 \mathrm{pg} / \mathrm{ml})$ e os sintomáticos tiveram seus níveis mais altos que todos os padrões de enchimento diastólico (fig. 4). A área abaixo da curva ROC do peptídeo natriurético tipo-B para detectar qualquer disfunção diastólica em pacientes com função sistólica normal foi de 0,92 (IC 95\% $0,87$ a $0.95 ; p<0.001)$. Esse foi o primeiro estudo em avaliar os diferentes padrões de enchimento ventricular esquerdo à ecocardiografia com Doppler e concluir que um teste-rápido de peptídeo natriurético tipo-B pode detectar com confiabilidade a presença de anormalidades diastólicas.

Esses estudos concluíram, todavia, que os níveis de peptídeo natriurético tipo-B, isoladamente, não podem di-

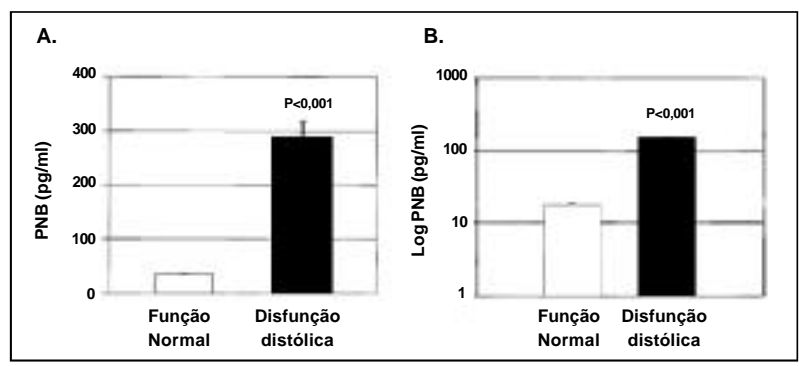

Fig. 4 - Níveis de peptídeo natriurético tipo-B em pacientes com função ventricular normal à ecocardiografia e com disfunção distólica. Dados estão expressos como média \pm erro padrão(A) e média logarítmica (B). Ambos significativas com $\mathrm{p}<0.001$. (Adaptado de Lubien e cols. ${ }^{15}$ ) ferenciar entre disfunção sistólica e diastólica e que um peptídeo natriurético tipo-B normal, num contexto de função sistólica normal, pode provavelmente descartar disfunção diastólica clinicamente significativa. De fato, para alguns pacientes, o achado de um peptídeo natriurético tipo-B normal pode dispensar a realização da ecocardiografia. Em pacientes com função sistólica normal e evidência clínica de insuficiência cardíaca, um nível elevado de peptídeo natriurético tipo-B pode substanciar o diagnóstico de disfunção diastólica.

\section{Peptídeo natriurético tipo-B e monitorização hemodinâmica na insuficiência cardíaca}

Oexame físico tem demonstrado limitada confiabilidade para indicar o perfil hemodinâmico de pacientes com insuficiência cardíaca crônica ${ }^{16,17}$. Um número de publicações tem sugerido que a "vasodilatação otimizada", com o objetivo de normalizar as pressões de enchimento ventricular esquerdo e a resistência vascular sistêmica em pacientes com insuficiência cardíaca e ventrículo dilatado, resultam em um melhor desfecho que o tratamento empírico ${ }^{18,19}$. Stevenson e cols. ${ }^{18}$ demonstraram, há mais de uma década, que pacientes com insuficiência cardíaca severa em lista de espera para transplante e capazes de responder à terapia otimizada, tinham uma mortalidade, de $38 \%$, em 1 ano, enquanto que os que não respondiam tinham uma mortalidade de $83 \%$, no mesmo período. Os benefícios da terapia otimizada com o auxílio do cateter de Swan Ganz reside na noção de que reduzir as pressões de enchimento ventricular para próximo do normal leva ao débito cardíaco ideal ${ }^{18}$, o que pode ocorrer não somente devido à redução da insuficiência mitral, comumente encontrada nesses pacientes, mas também por diminuir o consumo de oxigênio do miocárdio.

Entretanto, a abordagem da "vasodilatação otimizada" requer os cuidados de uma internação hospitalar em ambiente de tratamento intensivo e um procedimento invasivo para a inserção do cateter de Swan Ganz. Um simples exame de sangue, confiável em correlacionar com as alterações da pressão de enchimento ventricular esquerdo durante o manejo da insuficiência cardíaca, seria de grande valia.

Concentrações plasmáticas de peptídeo natriurético tipo-B não apenas refletem pressão de enchimento ventricular $^{20}$, como também estão fortemente correlacionadas às mudanças da pressão capilar pulmonar durante a hospitalização $(r=0.73, p<0.05)^{21}$. Pacientes que falharam à terapia com vasodilatação otimizada puderam ser identificados por meio de medidas de peptídeo natriurético tipo-B e eram, mais provavelmente, readmitidos ${ }^{21}$. Assim sendo, o peptídeo natriurético tipo-B pode ser um meio efetivo de melhorar o manejo intra-hospitalar de pacientes com insuficiência cardíaca severa, bem como permitir a possibilidade de uma "terapia otimizada ambulatorial", dispensando o auxílio do cateter de Swan Ganz.

\section{Peptídeo natriurético tipo-B efibrilação atrial}

Fibrilaçao atrial é uma doença freqüente entre idosos e/ ou pacientes com insuficiência cardíaca. É sabido que o 
peptídeo natriurético tipo-B é uma ferramenta não invasiva, seguramente correlacionada com pressão diastólica final do ventrículo esquerdo, sendo de fato um marcador de distensão miocárdica ${ }^{22}$. Estudos têm demonstrado que fibrilação atrial é um determinante independente de aumento dos níveis de fator natriurético atrial ${ }^{3}$. Embora o peptídeo natriurético tipo-B e, particularmente, o fator natriurético atrial, estejam supostamente elevados em pacientes com fibrilação atrial com ou sem disfunção ventricular, tem sido demonstrado que ambos os peptídeos tendem a diminuir após cardioversão elétrica bem sucedida ${ }^{23}$. A duração da fibrilação atrial também pode ter importância em pacientes com insuficiência cardíaca avançada, quanto mais longa a fibrilação atrial menores os níveis de fator natriurético atrial ${ }^{24}$. Outros autores têm demonstrado que, em pacientes com fibrilação atrial, o peptídeo natriurético tipo-B está aumentado e o miocárdio atrial considerado como fonte da sua produção ${ }^{25}$. Outra evidência da correlação entre arritmias, envolvendo dissincronia atrioventricular e níveis de peptídeo natriurético tipo-B, tem origem em um estudo que demonstrou níveis de peptídeo natriurético tipo-B mais elevados em pacientes com marcapasso em modo VVI do que aqueles em modos DDD ou $\mathrm{AAI}^{26}$. Após a cardioversão atrial, o peptídeo natriurético tipo-B diminui gradualmente ${ }^{23}$.

\section{Peptídeo natriurético tipo-B e cardiopatia isquêmica}

Um número significativo de estudos tem demonstrado que após infarto agudo do miocárdio, um elevado nível de peptídeo natriurético tipo-B está associado a infartos mais extensos $^{27}$, maior chance de remodelamento ventricular ${ }^{28}$, menor fração de ejeção ${ }^{29}$ e um maior risco de insuficiência cardíaca e morte ${ }^{30}$.

Mais recentemente, peptídeo natriurético tipo-B foi avaliado em todo o espectro das síndromes coronarianas agudas (SCA). Uma análise de subgrupos do estudo OPUS TIMI 16 revelou que níveis mais elevados de peptídeo natriurético tipo-B estavam associados com estenoses coronarianas $>50 \%$ e com testes de esforço positivo $(\mathrm{p}<0,001)^{31}$.

De Lemos e cols. ${ }^{32}$ relataram que, num contexto de síndrome coronariana aguda, um nível inicial elevado de peptídeo natriurético tipo-B esteve correlacionado com o risco de morte, insuficiência cardíaca ou infarto do miocárdio em 30 dias e 10 meses. Essa associação persistiu significativa em subgrupos de pacientes que apresentavam infarto agudo com elevação de segmento $S T(p<0,02)$, infarto sem elevação de ST ( $p<0,001)$ ou angina instável $(\mathrm{p}<0,001)$. Os sobreviventes tinham um peptídeo natriurético tipo-B médio de $80 \mathrm{pg} / \mathrm{ml}$ e os pacientes que morreram dentro de 30 dias após o infarto inicial tinham um peptídeo natriurético tipo-B médio de $153 \mathrm{pg} / \mathrm{ml}$ ( $\mathrm{p}<0,001)$. Esses achados foram independentes dos níveis de troponina, idade, presença ou ausência de insuficiência cardíaca, insuficiência renal ou alteração de segmento ST. Os autores concluíram que uma medida isolada de peptídeo natriurético tipo-B, obtida nos primeiros dias após o início de sintomas isquêmicos, teve po- der preditivo para ser usado na estratificação de risco de pacientes em todo o espectro das síndromes coronarianas agudas. A ativação neurohormonal cardíaca pode ser informação chave entre pacientes com alto risco de mortalidade após síndromes coronarianas agudas.

\section{Peptídeo natriurético tipo-B em procedimen- tos intervencionistas e síndromes coronaria- nas agudas.}

Peptídeo natriurético tipo-B também pode ser testado no contexto das intervenções coronarianas percutâneas. Elevados níveis de peptídeo natriurético tipo-B foram encontrados durante e imediatamente após angioplastia por balão, retornando à linha de base logo após o procedimento $^{33}$. Como consequiência, peptídeo natriurético tipo-B cresce em resposta ao aumento da pressão ventricular esquerda secundária à isquemia miocárdica transitória provocada pela angioplastia. A fim de confirmar esse suposto mecanismo, Tateishi e cols. ${ }^{34}$ estudaram um grupo de pacientes submetidos a cinecoronariografia diagnóstica e compararam com outro grupo submetido a intervenções coronarianas. Embora nenhuma alteração hemodinâmica tenha sido observada, antes e depois do procedimento, o peptídeo natriurético tipo-B plasmático esteve aumentado apenas no grupo dos pacientes submetidos à intervenção coronariana. Considerando esse resultado, o peptídeo natriurético tipo-B estaria possivelmente detectando mínimas alterações hemodinâmicas secundárias à isquemia induzida pelo balão que, na verdade, são indetectáveis pelas medidas hemodinâmicas convencionais. A medida da variação do peptídeo natriurético tipo-B durante angioplastia coronariana pode vir a ser um marcador bioquímico para indicar quanto do miocárdio isquêmico encontra-se em risco distalmente à lesão coronariana em questão.

Finalmente, de acordo com os resultados já mencionados, níveis séricos de peptídeo natriurético tipo-B foram definitivamente associados com risco aumentado de eventos cardíacos em 450 pacientes que apresentavam uma síndrome coronariana aguda. Em abordagem de estratificação de risco, baseada em múltiplos marcadores para a síndrome coronariana aguda, o peptídeo natriurético tipo-B demonstrou adicionar informação prognóstica independentemente dos outros marcadores ${ }^{35}$.

\section{Peptídeo natriurético tipo-B e diabetes mellitus}

É bem conhecido que pacientes diabéticos têm um risco aumentado de cardiopatia isquêmica e uma maior incidência de insuficiência cardíaca. Têm também uma maior prevalência tanto de disfunção sistólica como de diastólica do ventrículo esquerdo ${ }^{36}$. Essas anormalidades são freqüentemente assintomáticas, particularmente em pacientes diabéticos, embora possam ser tratadas com terapêuticas de eficácia comprovada, como inibidores da enzima conversora de angiotensina e betabloquadores ${ }^{37}$.

Apesar da ecocardiografia ser o primeiro exame para o 
diagnóstico de disfunção ventricular esquerda, ela tem significativas limitações nos pacientes diabéticos: não é prontamente disponível em clínicas para diabéticos, é usualmente reservada para pacientes sintomáticos e é de alto custo para teste de rastreamento, especialmente em pacientes assintomáticos. Entretanto, a identificação de pacientes diabéticos com disfunção ventricular esquerda é extremamente importante. Para fins de testar a hipótese de que o peptídeo natriurético tipo-B serviria como teste de rastreamento em pacientes diabéticos para a identificação de disfunção ventricular, Maisel e cols. ${ }^{38}$ estudaram 111 pacientes diabéticos encaminhados para ecocardiografia no Sistema de Saúde dos Veteranos de San Diego. Nos pacientes com função ventricular normal, peptídeo natriurético tipo-B foi de $39 \pm$ $8 \mathrm{pg} / \mathrm{ml}$, significativamente inferior aos pacientes com disfunção ventricular esquerda nos quais o peptídeo natriurético tipo-B foi de $379 \pm 138 \mathrm{pg} / \mathrm{ml}$, ou em pacientes com disfunção diastólica, onde foi de $479 \pm 106 \mathrm{pg} / \mathrm{ml}$. Pacientes com disfunção sistólica e diastólica apresentaram níveis de peptídeo natriurético tipo-B de $958 \pm 169 \mathrm{pg} / \mathrm{ml}$. A área sob a curva ROC demonstrando a sensibilidade e especificidade do peptídeo natriurético tipo-B contra o diagnóstico ecocardiográfico foi de 0,953 , concluindo que um teste rápido e simples, como o peptídeo natriurético tipo-B, pode ser empregado para rastrear de maneira confiável a presença ou ausência de disfunção ventricular em pacientes diabéticos, especialmente para detectar disfunção ventricular precoce em pacientes assintomáticos.

\section{Peptídeo natriurético tipo-B recombinante no tratamento da insuficiência cardíaca}

Finalmente, o peptídeo natriurético tipo-B tornou-se um exemplo de pesquisa evoluída da bancada do laboratório à beira do leito. Colucci e cols.$^{39}$ conduziram um ensaio-clínico randomizado para investigar o uso clínico de infusão intravenosa de nesiritide, um peptídeo natriurético tipo-B recombinante, em pacientes com insuficiência cardíaca descompensada, e demonstraram que o nesiritide é hemodi- namicamente benéfico e melhora significativamente os sintomas de insuficiência cardíaca, quando comparado ao placebo. No entanto, quando comparado ao tratamento convencional (com um agente vasoativo comumente empregado para tratamento a curto prazo de insuficiência cardíaca descompensada, como a dobutamina, milrinona, nitroglicerina ou nitroprussiato de sódio), nenhuma diferença significativa nos sintomas foi encontrada entre os grupos. Os autores concluiram que nesiritide é uma alternativa segura e uma importante associação ao tratamento inicial dos pacientes com insuficiência cardíaca descompensada.

\section{Conclusão}

O peptídeo natriurético tipo-B é um neurohormônio sintetizado no miocárdio atrial e ventricular e um indicador de pressões intracardíacas aumentadas. Pode estar aumentado devido a uma variedade de doenças cardíacas estruturais, como insuficiência cardíaca, disfunção sistólica ou diastólica, fibrilação atrial, isquemia aguda ou anormalidade valvular significativa. Neste sentido, o valor do peptídeo natriurético tipo-B reside no seu elevado valor preditivo negativo $(\approx 96 \%)$. No entanto, o peptídeo natriurético tipo-B vem se tornando: um teste de rastreamento para solicitações de ecocardiografia, independentemente da razão para o procedimento; uma ferramenta útil e confiável no diagnóstico e avaliação da severidade da insuficiência cardíaca na sala de emergência; um potencial marcador para monitorização hemodinâmica em pacientes com insuficiência cardíaca severa; um marcador prognóstico em pacientes que sofreram um infarto agudo do miocárdio.

Muitas outras aplicações estão ainda sob investigação e alguns autores ousam comparar a utilidade do peptídeo natriurético tipo-B para doenças cardiovasculares ao papel da creatinina para pacientes com doença renal. O peptídeo natriurético tipo-B intravenoso, como uma intervenção terapêutica, demonstrou-se tão efetivo quanto outras terapias convencionais.

\section{Referências}

1. De Bold AJ, Borenstein HB, Veress AT, Sonnenberg H. A rapid and potent natriuretic response to intravenous injection of atrial myocardial extract in rats. Life Sci 1981; 28:89-94.

2. Van D, Crijns HJ, Van V, Van G, De K, Lie KI. Atrial natriuretic peptide in patients with heart failure and chronic atrial fibrillation: role of duration of atrial fibrillation. Am Heart J 1998; 135:242-4.

3. Rossi A, Enriquez S, Burnett JC, Lerman A, Abel MD, Seward JB. Natriuretic peptide levels in atrial fibrillation: a prospective hormonal and Doppler-echocardiographic study. J Am Coll Cardiol 2000; 35:1256-62.

4. Wilkins MR, Redondo J, Brown LA. The natriuretic-peptide family. Lancet 1997; 349:1307-10.

5. Davis M, Espiner E, Richards G, et al. Plasma brain natriuretic peptide in assessment of acute dyspnoea. Lancet 1994; 343:440-4.

6. Dao Q, Krishnaswamy P, Kazanegra R, et al. Utility of B-type natriuretic peptide in the diagnosis of congestive heart failure in an urgent-care setting. J Am Coll Cardiol 2001; 37:379-85.

7. Harrison A, Morrison LK, Krishnaswamy P, et al. B-type natriuretic peptide predicts future cardiac events in patients presenting to the emergency department with dyspnea. Ann Emerg Med 2002; 39:131-8.

8. Maisel AS, Krishnaswamy P, Nowak RM, et al. Rapid Measurement of B-Type Natriuretic Peptide in the Emergency Diagnosis of Heart Failure. N Engl J Med 2002; 347:161-7.

9. Maisel AS, Krishnaswamy P, Nowak RM, et al. Rapid measurement of B-type natriuretic peptide in the emergency diagnosis of heart failure. N Engl J Med 2002; 347:161-7

10. Wong WF, Gold S, Fukuyama O, Blanchette PL. Diastolic dysfunction in elderly patients with congestive heart failure. Am J Cardiol 1989; 63:1526-8.

11. Bonow RO, Udelson JE. Left ventricular diastolic dysfunction as a cause of congestive heart failure: mechanisms and management. Ann Intern Med 1992; 117:502-10.

12. Krishnaswamy P, Lubien E, Clopton P, et al. Utility of B-natriuretic peptide levels in identifying patients with left ventricular systolic or diastolic dysfunction. Am J Med 2001; 111:274-9.

13. Lang CC, Prasad N, McAlpine HM, et al. Increased plasma levels of brain natriu- 
retic peptide in patients with isolated diastolic dysfunction. Am Heart J 1994; 127:1635-6.

14. Yamamoto K, Burnett JC, Jougasaki M, et al. Superiority of brain natriuretic peptide as a hormonal marker of ventricular systolic and diastolic dysfunction and ventricular hypertrophy. Hypertension 1996; 28:988-94.

15. Lubien E, DeMaria A, Krishnaswamy P, et al. Utility of B-natriuretic peptide in detecting diastolic dysfunction: comparison with Doppler velocity recordings. Circulation 2002; 105:595-601.

16. Stevenson LW, Perloff JK. The limited reliability of physical signs for estimating hemodynamics in chronic heart failure. JAMA 1989; 261:884-8

17. Rohde LE, Silva Neto LB, Lima MP, et al. Accuracy of clinical findings to determine hemodynamic status in outpatients with heart failure. J Card Fail 2001; 7:99

18. Stevenson LW, Tillisch JH, Hamilton M, et al. Importance of hemodynamic response to therapy in predicting survival with ejection fraction less than or equal to $20 \%$ secondary to ischemic or nonischemic dilated cardiomyopathy. Am J Cardiol 1990; 66:1348-54.

19. Stevenson LW. Tailored therapy to hemodynamic goals for advanced heart failure. Eur J Heart Fail 1999; 1:251-7.

20. Tsutamoto T, Wada A, Maeda K, et al. Attenuation of compensation of endogenous cardiac natriuretic peptide system in chronic heart failure: prognostic role of plasma brain natriuretic peptide concentration in patients with chronic symptomatic left ventricular dysfunction. Circulation 1997; 96:509-16.

21. Kazanegra R, Van Cheng BS, Garcia A, et al. A rapid test for B-type natriuretic peptide correlates with falling wedge pressures in patients treated for decompensated heart failure: a pilot study. J Card Fail 2001; 7:21-9.

22. Maeda K, Takayoshi T, Wada A. Plasma brain natriuretic peptide as a biochemical marker of high left ventricular end-diastolic pressure in patients with symptomatic left ventricular dysfunction. Am Heart J 1998; 135:825-32.

23. Ohta Y, Shimada T, Yoshitomi H, et al. Drop in plasma brain natriuretic peptide levels after successful direct current cardioversion in chronic atrial fibrillation. Can J Cardiol 2001; 17:415-20.

24. Van D, Tjeerdsma G, Jan d, Boomsma F, Crijns HJ, Van V. Longstanding atrial fibrillation causes depletion of atrial natriuretic peptide in patients with advanced congestive heart failure. Eur J Heart Fail 2002; 4:255-62.

25. Inoue SI, Murakami Y, Sano K, KatohH, Shimada T. Atrium as a source of brain natriuretic polypeptide in patients with atrial fibrillation. J Card Fail 2000; 6:92-6.

26. Horie H, Tsutamoto T, Ishimoto N. Plasma brain natriuretic peptide as a biochemical marker for atrioventricular sequence in patients with pacemakers. Pacing Clin Electrophysiol 1999; 22:282-90.
27. Arakawa N,Nakamura M,AokiH,Hiramori K. Relationship between plasma level of brain natriuretic peptide and myocardial infarct size. Cardiology 1994; 85:334-40.

28. Nagaya N, Nishikimi T, Goto Y, et al. Plasma brain natriuretic peptide is a biochemical marker for the prediction of progressive ventricular remodeling after acute myocardial infarction. Am Heart J 1998;135:21-8

29. Richards AM, Nicholls MG, Yandle TG, et al. Neuroendocrine prediction of left ventricular function and heart failure after acute myocardial infarction. The Christchurch Cardioendocrine Research Group. Heart 1999; 81:114-20.

30. Richards AM, Nicholls MG, Yandle TG, et al. Plasma N-terminal pro-brain natriuretic peptide and adrenomedullin: new neurohormonal predictors of left ventricular function and prognosis after myocardial infarction. Circulation 1998; 97:1921-9.

31. Cannon CP, McCabe CH, Wilcox RG, et al. Oral glycoprotein IIb/IIIa inhibition with orbofiban in patients with unstable coronary syndromes (OPUS-TIMI 16) trial. Circulation 2000; 102:149-56.

32. De Lemos JA, Morrow DA, Bentley JH, et al. The prognostic value of B-type natriuretic peptide in patients with acute coronary syndromes. N Engl J Med 2001;345:1014-21.

33. Kyriakides ZS, Markianos M, Michalis L, Antoniadis A, Nikolaou NI, Kremastinos DT. Brain natriuretic peptide increases acutely and much more prominently than atrial natriuretic peptide during coronary angioplasty. Clin Cardiol 2000; 23:285-8

34. Tateishi J, Masutani M, Ohyanagi M, Iwasaki T. Transient increase in plasma brain (B-type) natriuretic peptide after percutaneous transluminal coronary angioplasty. Clin Cardiol 2000; 23:776-80.

35. Sabatine MS, Morrow DA, de L, et al. Multimarker approach to risk stratification in non-ST elevation acute coronary syndromes: simultaneous assessment of troponin I, C-reactive protein, and B-type natriuretic peptide. Circulation 2002; 105:1760-3.

36. Annonu AK, Fattah AA, Mokhtar MS, Ghareeb S, Elhendy A. Left ventricular systolic and diastolic functional abnormalities in asymptomatic patients with noninsulin-dependent diabetes mellitus. J Am Soc Echocardiogr 2001; 14:885-91.

37. The SOLVD Investigattors. Effect of enalapril on mortality and the development. of heart failure in asymptomatic patients with reduced left ventricular ejection fractions. N Engl J Med 1992; 327:685-91.

38. Maisel AS, Morrison K, Demaria AN. The use of B-natriuretic peptide in assessing cardiac function in patients with diabetes mellitus. Circulation 2001; 104:2-3

39. Colucci WS, Elkayam U, Horton DP, et al. Intravenous nesiritide, a natriuretic peptide, in the treatment of decompensated congestive heart failure. N Engl J Med $2000 ; 343: 246-53$ 